\title{
Defining oligometastatic non-small cell lung cancer: concept versus biology, a literature review
}

\author{
Jill F. Mentink ${ }^{1}$, Marthe S. Paats ${ }^{1}$, Daphne W. Dumoulin ${ }^{1}$, Robin Cornelissen ${ }^{1}$, Joris B. W. Elbers ${ }^{2}$, \\ Alexander P. W. M. Maat ${ }^{3}$, Jan H. von der Thüsen ${ }^{4}$, Anne-Marie C. Dingemans ${ }^{1}$ \\ ${ }^{1}$ Department of Respiratory Medicine, Erasmus MC Cancer Institute, University Medical Center Rotterdam, Rotterdam, The Netherlands; \\ ${ }^{2}$ Department of Radiotherapy, Erasmus MC Cancer Institute, University Medical Center Rotterdam, Rotterdam, The Netherlands; ${ }^{3}$ Department \\ of Thoracic Surgery, Erasmus University Medical Center Rotterdam, Rotterdam, The Netherlands; ${ }^{4}$ Department of Pathology, Erasmus University \\ Medical Center Rotterdam, Rotterdam, The Netherlands \\ Contributions: (I) Conception and design: JF Mentink, MS Paats, AMC Dingemans; (II) Administrative support: None; (III) Provision of study \\ materials or patients: None; (IV) Collection and assembly of data: None; (V) Data analysis and interpretation: None; (VI) Manuscript writing: All \\ authors; (VII) Final approval of manuscript: All authors. \\ Correspondence to: Anne-Marie C. Dingemans, MD, PhD. Erasmus MC Cancer Institute, University Medical Center Rotterdam, department of \\ Respiratory Medicine, Dr. Molewaterplein 40, 3015 GD, Rotterdam, The Netherlands. Email: a.dingemans@erasmusmc.nl.
}

Objective: In this review, the concept of (synchronous) oligometastatic disease in patients with nononcogene-driven non-small cell lung cancer (NSCLC) will be placed in the context of tumor biology and metastatic growth patterns. We will also provide considerations for clinical practice and future perspectives, which will ultimately lead to better patient selection and oligometastatic disease outcome.

Background: The treatment landscape of metastasized NSCLC has moved from "one-size fits all" to a personalized approach. Prognosis has traditionally been poor but new treatment options, such as immunotherapy and targeted therapy, brighten future perspectives. Another emerging development is the recognition of patients with so-called "oligometastatic" state of disease. Oligometastatic disease has been recognized as a distinct clinical presentation in which the tumor is stated to be early in its evolution of metastatic potential. It is suggested that this stage of disease has an indolent course, comes with a better prognosis and therefore could be considered for radical multimodality treatment.

Methods: Narrative overview of the literature synthesizing the findings of literature retrieved from searches of computerized databases, hand searches, and authoritative texts.

Conclusions: Oligometastatic NSCLC is a broad spectrum disease, with a variable prognosis. Although the biology and behavior of "intermediate state" of metastatic disease are not fully understood, there is evidence that a subgroup of patients can benefit from local radical treatment when integrated into a multimodality regime. The consensus definition of oligometastatic NSCLC, including accurate staging, may help to uniform future trials. The preferable treatment strategy seems to sequential systemic treatment with subsequent local radical treatment in patients with a partial response or stable disease. Prognostic factors such as N-stage, number and site of distant metastases, tumor volume, performance status, age, and tumor type should be considered. The local radical treatment strategy has to be discussed in a multidisciplinary team meeting, taking into account patient characteristics and invasiveness of the procedure. However, many aspects remain to be explored and learned about the cancer biology and characteristics of intermediate state tumors.

Keywords: Lung cancer; oligometastatic; non-small cell lung cancer (NSCLC)

Submitted Apr 05, 2021. Accepted for publication Jun 10, 2021.

doi: $10.21037 /$ tlcr-21-265

View this article at: https://dx.doi.org/10.21037/tlcr-21-265 


\section{Introduction}

Worldwide, lung cancer is the leading cause of cancerrelated death in men and the second leading cause of death in women (1). Incidence and mortality are tightly linked to smoking, but numerous other risk factors have been identified (2). The five-year survival rates of non-small cell lung cancer (NSCLC) range from $80 \%$ for early-stage to $5-10 \%$ for metastatic disease (3). At presentation, $40 \%$ of patients have metastatic disease.

Metastatic NSCLC has traditionally been considered an incurable disease, with a median overall survival (OS) of less than one year (3). However, with the identification of oncogene-driven NSCLC and the introduction of tyrosine kinase inhibitors (TKIs), five-year survival rates have increased to $50 \%$ for this subgroup of patients $(4,5)$. Moreover, with the introduction of immune checkpoint inhibitors (ICIs), five-year survival now ranges from $15 \%$ in previously treated patients to $30 \%$ in first-line for patients with high PD-L1 expression (6). Next to these developments an emerging field of interest to improve outcome is identification of oligometastatic NSCLC, which has been recognized as a distinct clinical presentation in which the tumor is suggested to be early in its evolution of metastatic potential. In 1995, Hellman and Weichselbaum introduced the concept of oligometastatic disease as an "intermediate metastatic state" (7). This disease state is characterized by a more indolent course of disease, resulting in a better prognosis and therefore a potential for exploration of (radical) multimodality treatment. Cancer progression may thus not constitute an absolute divergence into non-metastatic disease which can be cured, and metastatic disease which cannot.

In this review, the concept of (synchronous) oligometastatic disease in NSCLC without driver aberrations will be evaluated in the context of tumor biology and metastatic growth patterns. We will also provide considerations for clinical practice and future perspectives, which may ultimately lead to better patient selection and outcomes in oligometastatic disease.

We present the following article in accordance with the Narrative Review reporting checklist (available at https:// dx.doi.org/10.21037/tlcr-21-265).

\section{Methods}

Narrative overview of the literature synthesizing the findings of literature retrieved from searches of PubMed.
Only publication in English language were selected.

\section{Defining oligometastatic disease}

Oligometastatic disease is considered a clinical stage with a limited number of metastases in a limited number of organs, possibly reflecting an "intermediate state of metastasis" (7). For these patients radical treatment of all tumor sites might be a meaningful intervention resulting in long term OS. Regarding NSCLC, there is increasing consensus and some recent evidence to support this concept (8-11). The landmark study of Gomez et al. showed a clear benefit for local radical therapy versus maintenance therapy or observation in patients with NSCLC with a low metastatic burden who did not progress after front line systemic therapy (9). The study was closed prematurely because of a significant median progression free survival (PFS) advantage of 11.9 months [90\% confidence interval (CI), 5.7-20.9] versus 3.9 months (90\% CI, 2.3-6.6), with a hazard ratio (HR) of 0.35 (90\% CI, 0.18-0.66, $\mathrm{P}=0.0054$ ). Also, a significant OS benefit in the treatment arm was seen with a median of 41.2 months (95\% CI, 18.9-not reached) versus 17 months (95\% CI, $10.1-39.8, \mathrm{P}=0.017$ ) in the control arm (11). As this study was closed pre-maturely these results are based on only 48 patients, of whom 8 had oncogene driven NSCLC. In order to be eligible, up to three non-progressing metastatic lesions after frontline systemic treatment were allowed. Brain imaging was mandatory, however fluorodeoxyglucose (FDG) positron emission tomography (PET) computed tomography imaging (CT) was not. With this trial in mind the definition of oligometastatic NSCLC will be discussed.

It has been estimated that approximately $50 \%$ of patients with metastatic NSCLC present with a maximum of three metastatic sites (12). The accuracy of cancer staging has been improved by the introduction of more sensitive imaging techniques, FDG-PET-CT and magnetic resonance imaging (MRI). Approximately $15 \%$ of patients initially classified as stage I-III NSCLC will be upstaged to stage IV after performing an FDG-PET CT $(13,14)$. Preoperative FDG-PET-CT staging significantly improved five-year OS (58\% versus 33\%) in surgically resected NSCLC compared to conventional CT-staging (14). This can be explained by the "Will Rogers phenomenon", defined as an improved overall outcome despite unaltered individual prognoses due to stage migration (15). Stage migration is essential in the concept of oligometastatic 
NSCLC: by more accurate and sensitive staging methods such as FDG-PET-CT, we will identify more patients with this transitional state between strictly localized and widespread systemic disease. This comes with an element of uncertainty because some of these patients might still have a rapidly progressing disease that cannot be distinguished from the real oligometastatic state.

One of the major controversies is the maximum number of metastases that can be considered "oligo" metastatic. Most studies of oligometastatic NSCLC to date allow up to five metastases, however in reality the majority of patients included in these studies had a maximum of up to three metastases $(16,17)$.

In the 8th TNM implemented in 2018 patients with metastases were subdivided into three categories of prognostic relevance: patients with only lung involvement (M1a), versus a single extra-thoracic organ metastasis (M1b), versus multiple extra-thoracic metastasis in one or more organs (M1c) (18). This reclassification reflects the differences in median survival. For M1a this is 22.5 months, in M1b 17.7 months and in M1c 13.6 months (19). Although this identifies patients with a solitary extrapulmonary metastasis, this is not a definition of oligometastastic NSCLC. The European Organization of Research and Treatment of Cancer-Lung Cancer Group (EORTC-LCG) has published a consensus statement on the definition of oligometastatic NSCLC (20). This goal of this statement was the provision of a uniform definition and staging of synchronous oligometastatic disease in NSCLC. It highlights that the concept of oligometastatic treatment should only be used when "a radical treatment that may modify the disease course (leading to long-term disease control) is technically feasible for all tumor sites with acceptable toxicity". Histology, genomic background and tumor volume are not taken into account in the definition. A maximum of 5 metastases in 3 organs is proposed, but diffuse serosal (meningeal, pleural, pericardial, and mesenteric) or bone marrow metastases are excluded from this definition. Also noteworthy is that a pulmonary metastasis should be seen as a metastatic site. Mediastinal lymph nodes are considered loco-regional disease and are essential in determining whether or not radical treatment of the primary tumor can be applied. In the consensus statement, the importance of staging is stressed and both FDG-PET-CT and imaging of the brain are mandatory for the staging of oligometastatic NSCLC. This is in line with advice from the EORTCimaging group (21).

\section{Perspectives on the different oligometastatic states}

Four different states of oligometastatic disease in NSCLC can be distinguished (22). First is "synchronous oligometastatic disease", in which at initial work up one or more metastases are found. Secondly, "metachronous oligometastatic disease" is considered a limited relapse after treatment with curative intention. Third is "oligopersistent disease" or "oligoresidual disease", reflecting oligometastatic disease after initial treatment. Fourthly the term "oligoprogression" is used if over time only a few metastatic sites progress. For the purpose of this review we will mainly focus on this type of oligometastatic disease.

In synchronous oligometastatic disease more than onethird of the patients who were able to complete treatment with radical intent on both primary and metastatic sites, have a two-year survival similar to the stage III NSCLC patient population who are treated with definitive chemoradiotherapy (23). Although this would appear to represent promising long term survival, there is an unfavorable subgroup of patients that progress during treatment. Moreover, most patients tend to progress within a short period, which suggests the presence of micrometastases at the time of treatment (24). Therefore, an acceptable multimodality strategy could be to initiate systemic treatment and re-stage the patient before initiating local radical treatment for the primary tumor and metastasis, as exemplified by the study design of Gomez et al. (8-10). Another option is to start with radical treatment of metastases followed by systemic treatment. However, results following this approach are poor and possibly explained by the lack of adequate patient selection (24).

\section{Prognostic factors in oligometastatic NSCLC}

As stated before, it seems that not all patients with oligometastatic NSCLC have long term benefit from local radical treatment. There have been multiple, mainly retrospective, studies focusing on prognostic factors in oligometastastic NSCLC. A systematic review, published in 2013, retrieved 23 studies (on total 1,064 patients) in which a multivariable analysis for prognostic factors for survival of patients with metastatic NSCLC and up to five metastases showed that $\mathrm{N}$-stage was a highly significant prognostic factor (25). More recently, Frost et al. conducted a retrospective study to identify prognostic factors for survival in oligometastatic NSCLC (26). They found 
that a limited primary tumor size (T1a), adenocarcinoma histology, low nodal status (N0, N1 versus N2), a low number of metastases (especially solitary metastases), and good performance status (ECOG 0 or 1 ) were associated with a better outcome. This was confirmed in a prospective study by Parikh et al., which showed that poor performance status (ECOG $\geq 2$ ), advanced nodal status (N2 or N3), squamous histology and metastases to multiple organs were associated with a higher HR of death (27). In NSCLC in general, favorable outcomes have been described in patients with skip lymph nodes metastases. The five-year OS of patients with pN2 but negative N1 was found to be $41 \%$ compared to only $14 \%$ in patients with $\mathrm{pN} 2$ in addition to N1 involvement (28). In a large cohort of patients with stage M1a NSCLC from the Surveillance Epidemiology and End Results (SEER) program, nodal status had an impact on survival, as patients with N0-1 status in particular had a better outcome (29). Unfortunately, only one out of six patients with metastatic NSCLC present with N0 status (30). In $25.3 \%$ of patients with resected lung cancer micrometastases are detected in lymph nodes by additional analysis (e.g., multiple-level sectioning, additional immunohistochemical and molecular biological techniques) (31). This is currently not implemented in daily clinical practice as it has no therapeutical consequences. However, such additional techniques could lead to better prognostic stratification in patients with oligometastatic NSCLC. In patients with an isolated adrenal gland metastasis who underwent adrenalectomy, 5-year OS in N0 disease was $53 \%$ compared to $0 \%$ when mediastinal lymph node metastases are present (32).

In addition to nodal involvement, other factors seem relevant to outcome, including the site of extra-thoracic metastases and tumor volume. In particular, data from a small retrospective series indicate that lesions in the brain or adrenal glands are associated with prolonged survival compared with bone or liver metastases (33). A systematic review on solitary metastases did not show differences in survival between less common metastatic sites such as bone, liver, spleen, and kidney (34). Also, satisfactory outcomes have been reported for pancreatic metastases, albeit in small cohorts (35).

Several studies have shown that tumor volumes can also hold prognostic value. Thus, gross primary tumor volume was associated with OS (36), while another study showed that larger tumor volumes were predictive of high rates of local failure (37). In this light, T stage upon initiation of treatment needs to be taken into account for treatment planning.

\section{Cancer biology}

To achieve a better understanding of the oligometastatic state, we need to consider the biology of cancer in general. This might help to select patients with an actual intermediate state of disease. The original well-known six hallmarks of cancer provide a widely accepted insight into carcinogenesis (38). The first hallmark is the capacity of the cancer cell to initiate and sustain proliferative signaling: in normal tissue the cellular growth and division cycle is carefully controlled, but cancer cells can deregulate growth signals and thereby sustain chronic proliferation. Evading the influence of growth suppressors is an important second hallmark. A dozen tumor suppressors negatively regulate cell growth, including the RB and TP53 proteins, which act as critical gatekeepers and govern the cellular switch to either continue proliferation or activate senescence and apoptosis. Third, cancer cells can resist forms of cell death which are typically induced by anticancer therapy or physiological stress. Fourth, the acquisition of replicative immortality is necessary to generate a macroscopic tumor; this characteristic contrasts with normal cell lineages that can only undergo a limited number of successive growth cycles. Fifth is the capacity to induce angiogenesis, which is essential to match tumoral oxygen and nutrient demand and evacuate metabolic waste. The sixth hallmark is the physical dissemination of cancer cells from the primary tumor and the ensuing spread to distant organs. This involves processes of loss of cellular adhesion, epithelialto-mesenchymal transition (EMT), and degradation of extracellular matrix by matrix metalloproteases (MMPs), as initiated by alteration of E-cadherin/ $\beta$-catenin signaling and activation of transcription by the transcription factors Twist, Snail, Slug, and ZEB $(39,40)$. These processes are also crucial for distant colonization, including adaptation to the new microenvironment (including a reverse process of mesenchymal-to epithelial transition), following which micrometastases can grow and form a distant macroscopic tumor. This is a trial and error process and explains why years after radical treatment of the primary tumor, distant metastasis can still occur. In an update of the paradigm, reprogramming of cellular metabolism and avoidance of immune destruction were added to the known hallmarks (41). It is noteworthy that underlying these hallmarks are (I) the enabling characteristics of genome instability, which give cancer cells the ability to mutate, and (II) the inflammatory 
state driven by our own immune system that, unfortunately, can also sustain a tumor-promoting inflammatory environment.

That being said, the oncogenic transformation and pathways of spread are only partially understood, and metastatic spread in general remains a challenging problem in NSCLC. A series of interactions ranging from alteration and escape of the tumor microenvironment, entry and survival in the circulation, seeding and invasion into a distant site, and finally adaptation to, or development of, the appropriate microenvironment are necessary before distant organ colonization can occur (42). Only a small proportion of tumor cells that escape the primary tumor ultimately colonize a distant organ site (43)

More than a century ago the "seed and soil" theory was introduced by Paget (44), which suggested that tumor cells (seeds) selectively (and not randomly) colonize compatible distant organs (soil). Various animal studies have tested and confirmed this concept (45-47). It has become clear that metastases do not solely depend on trapping and lodging tumor cells from the bloodstream to colonize distant organs. Each organ is variable in its accessibility, stromal characteristics, amounts of vascular and nutrient supply (43). Numerous growth factors, chemokines, guidance molecules, signaling pathways and genes have been discovered to be involved (48). Shortfalls in this metastatic cascade could result in a phenotype with limited metastatic potential (49), a particular example of which is metastatic tumor dormancy. According to this concept, disseminated tumor cells arrive at a secondary site but stop proliferating and remain quiescent for an extended latency (50). Maintaining a state of tumor dormancy has the potential to transform cancer into a chronic disease, although it is preferable to attempt elimination of all cancer cells because of the risk of proliferation under the influence of new stimuli (51).

A possible biological explanation for the origin of oligometastases is that there may be primary tumor cells with a limited capacity regarding one or more of the required biological hallmarks for metastasis (7). However, to which extent the metastatic potential and possible dormancy are already deterministically enshrined in phylogenetic subgroups defined by different combinations and sequences of genetic alterations, remains to be resolved for NSCLC (52). Nevertheless, this could offer the possibility of subtyping tumors according to their likely future metastatic patterns, and thus suitability for oligometastatic treatment approaches.

\section{Clinical considerations}

Although we are still searching for a better understanding of the oligometastatic concept, it is currently being applied in daily clinical practice using local radical therapies aimed at long-term disease control or possibly even curation. The most pivotal question is which patients truly present with an intermediate metastatic state of disease and thus benefit from oligometastatic-based treatment. Therefore, it is advisable to first evaluate tumor response to systemic treatment before initiating local consolidative treatment, conform the concept of the Gomez trial (9).

The type of radical treatment is also important to consider. Nowadays (minimally invasive) surgery, stereotactic radiotherapy and cryo-ablation are examples of treatment options with acceptable safety profiles. Weighing all options, in particular the site of disease, performance status of the patient and recovery time (which might delay initiation of treatment) play a pivotal role. There is some evidence suggesting that surgery, or even minor trauma such as a biopsy, can influence disease course in a way that leads to unfavorable outcomes (53). It has been suggested that this is related to pathophysiological processes that enhance postoperative tumor spread, which lead to recurrent disease. These mechanisms can cause a paradoxical growth of metastases after surgical intervention. However, others have reported that in NSCLC with a solitary extrathoracic synchronous metastasis, surgical metastasectomy was not prognostically better or worse than radiotherapy with or without chemotherapy (54). Stereotactic radiotherapy is feasible in selected patients and well-tolerated (55). Another potential advantage of radiotherapy is the induction of the so-called "abscopal effect" (56). The abscopal effect is a rare phenomenon in which the regression of tumor lesions outside the treated volume is seen. There is evidence of enhanced anti-tumor immunity via $\mathrm{CD} 8+\mathrm{T}$-cells and cellular stress signals after high-dose, hypofractionated radiation. It is believed to be a relatively uncommon phenomenon in lung cancer due to its inherent low immunogenicity. The proposed mechanism is that radiotherapy stimulates the immune system by releasing anti-tumor proliferative agents and cytokines (57). It is thought that the tumor and the immune system of the host are in a constant fight in which, on the one hand of the spectrum, (I) tumor is eliminated, through (II) an equilibrium of subclinical tumor ("tumor dormancy"), to (III) resistance and evolution of a clinically detectable tumor 
mass on the other end of the spectrum. Therefore, apart from local benefit, an emerging advantage of stereotactic ablative body radiation may be fortification of the immune system. Additional immunotherapy treatment might have a further synergistic effect (58). SBRT releases neo-antigens and stimulates maturation and proliferation of naive T-cells, while immunotherapy activates and amplifies those naive T-cells, enhancing tumoricidal effects (59).

\section{Future perspectives}

More sensitive prognostic staging and the identification of additional biomarkers (e.g., tumor-genetic profiles, circulating cell-free DNA (cfDNA) or circulating tumor cells) in combination with classical (Bayesian) modeling or artificial intelligence will allow future upfront selection of the patient population that will benefit from this approach. This is especially important because the direct initiation of local radical treatment will result in overtreatment in a subgroup of patients.

Emerging opportunities can be found in the optimization of staging afforded by improvement and availability of radiology techniques. This will enable a more thorough selection of patients with a low metastatic burden in whom multimodality treatment can be applied to all metastatic sites.

Circulating tumor DNA (ctDNA) analysis has revolutionized care in patients on targeted treatment $(60,61)$. In the Gomez trial decrease in ctDNA was observed in the patients treated with local consolidative treatment (62).

In-depth tumor-genetic analysis could also hold prognostic value. For example, in breast cancer several genes have been identified that seem to play a role in development of site-specific metastases (63). Another promising tool is micro-RNA, which are small noncoding RNA strands of which some can regulate tumor proliferation and apoptosis, and in pre-clinical studies some micro-RNAs appear to mediate a metastatic phenotype (64). This can help to identify more rapidly progressive tumors in which oligometastatic treatment has no additional value. Micro-RNAs can also be used as additional staging tool (65). Furthermore, investigations have found micro-RNAs that suppressed crucial steps in the formation of distant organ colonization, such as cellular adhesion and invasion (66). Ultimately, if micro-RNAs could be targeted, this could hold great potential for limiting the extent of metastasis.

It is also important to continue the study of cancer biology, including tumor stroma heterogeneity and the role of micro-environmental factors which modulate therapy resistance (67). In this light, the role of local radical therapy combined with immunotherapy and targeted therapies should also be investigated.

\section{Conclusions}

Oligometastatic NSCLC is a broad spectrum disease, with a variable prognosis. Although the biology and behavior of "intermediate state" of metastatic disease are not fully understood, there is evidence that a subgroup of patients can benefit from local radical treatment when integrated into a multimodality regime. Furthermore, until recently, there was no clear definition of oligometastatic disease in NSCLC, but a consensus definition has now been developed. This definition relies on accurate staging with improved imaging techniques. Currently, there are no biomarkers or other techniques that can reliably identify patients with oligometastatic disease who are in a true oligo-metastatic state.

A promising strategy is to first monitor tumor behavior during initial systemic treatment, followed by reassessment after four cycles. The advantages of this approach are the reduction of tumor burden in a short period of time and the selection of patients who rapidly progress and are thus unlikely to benefit from local radical treatment. Subsequent local radical treatment in synchronous oligometastatic disease seems desirable if there is a partial response or stable disease under systemic treatment. Prognostic factors such as $\mathrm{N}$-stage, number and site of distant metastases, tumor volume, performance status, age, and tumor type should be considered. The local radical treatment strategy has to be discussed in a multidisciplinary team meeting, taking into account patient characteristics and invasiveness of the procedure. A limitation of this review is that we did not perform a systematic review. However, this is not feasible as we did not have one research question.

While the time trend is already showing improved OS in oligometastatic NSCLC treated with radical intent, an effect that we might be able to enhance in the near future, many aspects remain to be explored and learned about the cancer biology and characteristics of intermediate state tumors. In this way, we can further define the concept of oligometastatic disease and develop and implement novel treatment strategies.

\section{Acknowledgments}

Funding: None. 


\section{Footnote}

Provenance and Peer Review: This article was commissioned by the Guest Editors (Maurizio Infante \& Thierry Berghmans) for the series "Oligometastatic NSCLC: definition and treatment opportunities" published in Translational Lung Cancer Research. The article has undergone external peer review.

Reporting Checklist: The authors have completed the Narrative Review reporting checklist. Available at https:// dx.doi.org/10.21037/tlcr-21-265

Conflicts of Interest: All authors have completed the ICMJE uniform disclosure form (available at https://dx.doi. org/10.21037/tlcr-21-265). The series "Oligometastatic NSCLC: definition and treatment opportunities" was commissioned by the editorial office without any funding or sponsorship. DWD reports consulting fees from Roche, MSD, Novartis, Pfizer, Astra Zeneca, BMS, outside the submitted work. RC reports speaker fee from Roche, Pfizer and BMS, personal fees from Advisory board of MSD, Roche and Spectrum, outside the submitted work. AMCD reports personal fees from Roche, eli Lilly, Boehringer Ingelheim, Astra Zeneca, Pfizer, BMS, Amgen, Novartis, Takeda, Pharmamar and MSD, grants from BMS and Amgen, non-financial support from Abbvie, outside the submitted work. The authors have no other conflicts of interest to declare.

Ethical Statement: The authors are accountable for all aspects of the work in ensuring that questions related to the accuracy or integrity of any part of the work are appropriately investigated and resolved.

Open Access Statement: This is an Open Access article distributed in accordance with the Creative Commons Attribution-NonCommercial-NoDerivs 4.0 International License (CC BY-NC-ND 4.0), which permits the noncommercial replication and distribution of the article with the strict proviso that no changes or edits are made and the original work is properly cited (including links to both the formal publication through the relevant DOI and the license). See: https://creativecommons.org/licenses/by-nc-nd/4.0/.

\section{References}

1. Sung H, Ferlay J, Siegel RL, et al. Global cancer statistics
2020: GLOBOCAN estimates of incidence and mortality worldwide for 36 cancers in 185 countries. CA Cancer J Clin 2021;71:209-49.

2. Barta JA, Powell CA, Wisnivesky JP. Global Epidemiology of Lung Cancer. Ann Glob Health 2019;85:8.

3. Goldstraw P, Chansky K, Crowley J, et al. The IASLC Lung Cancer Staging Project: Proposals for Revision of the TNM Stage Groupings in the Forthcoming (Eighth) Edition of the TNM Classification for Lung Cancer. J Thorac Oncol 2016;11:39-51.

4. Solomon BJ, Kim DW, Wu YL, et al. Final Overall Survival Analysis From a Study Comparing First-Line Crizotinib Versus Chemotherapy in ALK-MutationPositive Non-Small-Cell Lung Cancer. J Clin Oncol 2018;36:2251-8.

5. Yamamoto N, Seto T, Nishio M, et al. Erlotinib plus bevacizumab vs erlotinib monotherapy as first-line treatment for advanced EGFR mutation-positive nonsquamous non-small-cell lung cancer: Survival follow-up results of the randomized JO25567 study. Lung Cancer 2021;151:20-4.

6. Garon EB, Hellmann MD, Rizvi NA, et al. Five-Year Overall Survival for Patients With Advanced NonSmallCell Lung Cancer Treated With Pembrolizumab: Results From the Phase I KEYNOTE-001 Study. J Clin Oncol 2019;37:2518-27.

7. Hellman S, Weichselbaum RR. Oligometastases. J Clin Oncol 1995;13:8-10.

8. Iyengar P, Wardak Z, Gerber DE, et al. Consolidative Radiotherapy for Limited Metastatic Non-Small-Cell Lung Cancer: A Phase 2 Randomized Clinical Trial. JAMA Oncol 2018;4:e173501.

9. Gomez DR, Blumenschein GR Jr, Lee JJ, et al. Local consolidative therapy versus maintenance therapy or observation for patients with oligometastatic non-smallcell lung cancer without progression after first-line systemic therapy: a multicentre, randomised, controlled, phase 2 study. Lancet Oncol 2016;17:1672-82.

10. Gomez DR, Tang C, Zhang J, et al. Local Consolidative Therapy (LCT) Improves Overall Survival (OS) Compared to Maintenance Therapy/Observation in Oligometastatic Non-Small Cell Lung Cancer (NSCLC): Final Results of a Multicenter, Randomized, Controlled Phase 2 Trial. International Journal of Radiation Oncology Biology Physics 2018;102:1604.

11. Gomez DR, Tang C, Zhang J, et al. Local Consolidative Therapy Vs. Maintenance Therapy or Observation for Patients With Oligometastatic Non-Small-Cell Lung 
Cancer: Long-Term Results of a Multi-Institutional, Phase II, Randomized Study. J Clin Oncol 2019;37:1558-65.

12. Mehta N, Mauer AM, Hellman S, et al. Analysis of further disease progression in metastatic non-small cell lung cancer: implications for locoregional treatment. Int J Oncol 2004;25:1677-83.

13. Dinan MA, Curtis LH, Carpenter WR, et al. Stage migration, selection bias, and survival associated with the adoption of positron emission tomography among medicare beneficiaries with non-small-cell lung cancer, 1998-2003. J Clin Oncol 2012;30:2725-30.

14. Tonnies S, Tonnies M, Kollmeier J, et al. Impact of preoperative 18F-FDG PET/CT on survival of resected mono-metastatic non-small cell lung cancer. Lung Cancer 2016;93:28-34.

15. Feinstein AR, Sosin DM, Wells CK. The Will Rogers phenomenon. Stage migration and new diagnostic techniques as a source of misleading statistics for survival in cancer. N Engl J Med 1985;312:1604-8.

16. Giaj-Levra N, Giaj-Levra M, Durieux V, et al. Defining Synchronous Oligometastatic Non-Small Cell Lung Cancer: A Systematic Review. J Thorac Oncol 2019;14:2053-61.

17. Giaj-Levra N, Giaj Levra M, Berghmans T, et al. Oligometastatic non-small cell lung cancer (NSCLC): Does number of metastasis matter? Lung Cancer 2020;139:216-8.

18. Eberhardt WEE, Mitchell A, Crowley J, et al. The IASLC Lung Cancer Staging Project Proposals for the Revision of the M Descriptors in the Forthcoming Eighth Edition of the TNM Classification of Lung Cancer. J Thorac Oncol 2015;10:1515-22.

19. Shin J, Keam B, Kim M, et al. Prognostic Impact of Newly Proposed M Descriptors in TNM Classification of Non-Small Cell Lung Cancer. J Thorac Oncol 2017;12:520-8.

20. Dingemans AC, Hendriks LEL, Berghmans T, et al. Definition of Synchronous Oligometastatic Non-Small Cell Lung Cancer-A Consensus Report. J Thorac Oncol 2019;14:2109-19.

21. deSouza NM, Liu Y, Chiti A, et al. Strategies and technical challenges for imaging oligometastatic disease: Recommendations from the European Organisation for Research and Treatment of Cancer imaging group. Eur J Cancer 2018;91:153-63.

22. Guckenberger M, Lievens Y, Bouma AB, et al. Characterisation and classification of oligometastatic disease: a European Society for Radiotherapy and
Oncology and European Organisation for Research and Treatment of Cancer consensus recommendation. Lancet Oncol 2020;21:e18-e28.

23. Griffioen GH, Toguri D, Dahele M, et al. Radical treatment of synchronous oligometastatic non-small cell lung carcinoma (NSCLC): patient outcomes and prognostic factors. Lung Cancer 2013;82:95-102.

24. De Ruysscher D, Wanders R, Hendriks LE, et al. Progression-Free Survival and Overall Survival Beyond 5 Years of NSCLC Patients With Synchronous Oligometastases Treated in a Prospective Phase II Trial (NCT 01282450). J Thorac Oncol 2018;13:1958-61.

25. Ashworth A, Rodrigues G, Boldt G, et al. Is there an oligometastatic state in non-small cell lung cancer? A systematic review of the literature. Lung Cancer 2013;82:197-203.

26. Frost N, Tessmer A, Schmittel A, et al. Local ablative treatment for synchronous single organ oligometastatic lung cancer-A propensity score analysis of 180 patients. Lung Cancer 2018;125:164-73.

27. Parikh RB, Cronin AM, Kozono DE, et al. Definitive primary therapy in patients presenting with oligometastatic non-small cell lung cancer. Int J Radiat Oncol Biol Phys 2014;89:880-7.

28. Prenzel KL, Monig SP, Sinning JM, et al. Role of skip metastasis to mediastinal lymph nodes in non-small cell lung cancer. J Surg Oncol 2003;82:256-60.

29. Dai C, Ren Y, Xie D, et al. Does Lymph Node Metastasis Have a Negative Prognostic Impact in Patients with NSCLC and M1a Disease? J Thorac Oncol 2016;11:1745-54.

30. Tamura T, Kurishima K, Watanabe H, et al. Characteristics of clinical N0 metastatic non-small cell lung cancer. Lung Cancer 2015;89:71-5.

31. Jeong JH, Kim NY, Pyo JS. Prognostic roles of lymph node micrometastasis in non-small cell lung cancer. Pathol Res Pract 2018;214:240-4.

32. Raz DJ, Lanuti M, Gaissert HC, et al. Outcomes of patients with isolated adrenal metastasis from non-small cell lung carcinoma. Ann Thorac Surg 2011;92:1788-92; discussion 1793.

33. Divisi D, Barone M, Zaccagna G, et al. Surgical approach in the oligometastatic patient. Ann Transl Med 2018;6:94.

34. Salah S, Tanvetyanon T, Abbasi S. Metastatectomy for extra-cranial extra-adrenal non-small cell lung cancer solitary metastases: systematic review and analysis of reported cases. Lung Cancer 2012;75:9-14. 
35. DeLuzio MR, Moores C, Dhamija A, et al. Resection of oligometastatic lung cancer to the pancreas may yield a survival benefit in select patients--a systematic review. Pancreatology 2015;15:456-62.

36. Lopez Guerra JL, Gomez D, Zhuang Y, et al. Prognostic impact of radiation therapy to the primary tumor in patients with non-small cell lung cancer and oligometastasis at diagnosis. Int J Radiat Oncol Biol Phys 2012;84:e61-7.

37. Merino Lara T, Helou J, Poon I, et al. Multisite stereotactic body radiotherapy for metastatic non-smallcell lung cancer: Delaying the need to start or change systemic therapy? Lung Cancer 2018;124:219-26.

38. Hanahan D, Weinberg RA. The hallmarks of cancer. Cell 2000;100:57-70.

39. Ancel J, Dewolf M, Deslee G, et al. Clinical Impact of the Epithelial-Mesenchymal Transition in Lung Cancer as a Biomarker Assisting in Therapeutic Decisions. Cells Tissues Organs 2020. doi: 10.1159/000510103.

40. Sung WJ, Kim H, Park KK. The biological role of epithelial-mesenchymal transition in lung cancer (Review). Oncol Rep 2016;36:1199-206.

41. Hanahan D, Weinberg RA. Hallmarks of Cancer: The Next Generation. Cell 2011;144:646-74.

42. Gupta GP, Massague J. Cancer metastasis: Building a framework. Cell 2006;127:679-95.

43. Obenauf AC, Massague J. Surviving at a Distance: OrganSpecific Metastasis. Trends Cancer 2015;1:76-91.

44. Paget $\mathrm{S}$. The distribution of secondary growths in cancer of the breast. 1889. Cancer Metastasis Rev 1989;8:98-101.

45. Sugarbaker ED. The organ selectivity of experimentally induced metastases in rats. Cancer 1952;5:606-12.

46. Kinsey DL. An experimental study of preferential metastasis. Cancer 1960;13:674-6.

47. Hart IR, Fidler IJ. Role of organ selectivity in the determination of metastatic patterns of B16 melanoma. Cancer Res 1980;40:2281-7.

48. Fokas E, Engenhart-Cabillic R, Daniilidis K, et al. Metastasis: the seed and soil theory gains identity. Cancer Metastasis Rev 2007;26:705-15.

49. Corbin KS, Hellman S, Weichselbaum RR. Extracranial Oligometastases: A Subset of Metastases Curable With Stereotactic Radiotherapy. Journal of Clinical Oncology 2013;31:1384-90.

50. Neophytou CM, Kyriakou TC, Papageorgis P. Mechanisms of Metastatic Tumor Dormancy and Implications for Cancer Therapy. Int J Mol Sci 2019;20:6158.
51. Altorki NK, Markowitz GJ, Gao DC, et al. The lung microenvironment: an important regulator of tumour growth and metastasis. Nat Rev Cancer 2019;19:9-31.

52. Turajlic S, Xu H, Litchfield K, et al. Deterministic Evolutionary Trajectories Influence Primary Tumor Growth: TRACERx Renal. Cell 2018;173:595-610.e11.

53. Tohme S, Simmons RL, Tsung A. Surgery for Cancer: A Trigger for Metastases. Cancer Res 2017;77:1548-52.

54. Mordant P, Arame A, De Dominicis F, et al. Which metastasis management allows long-term survival of synchronous solitary M1b non-small cell lung cancer? Eur J Cardiothorac Surg 2012;41:617-22.

55. Schanne DH, Heitmann J, Guckenberger M, et al. Evolution of treatment strategies for oligometastatic NSCLC patients - A systematic review of the literature. Cancer Treat Rev 2019;80:101892.

56. Garelli E, Rittmeyer A, Putora PM, et al. Abscopal effect in lung cancer: three case reports and a concise review. Immunotherapy 2019;11:1445-61.

57. Lee Y, Auh SL, Wang Y, et al. Therapeutic effects of ablative radiation on local tumor require CD8+ $\mathrm{T}$ cells: changing strategies for cancer treatment. Blood 2009;114:589-95.

58. Theelen WSME, Peulen HMU, Lalezari F, et al. Effect of Pembrolizumab After Stereotactic Body Radiotherapy vs Pembrolizumab Alone on Tumor Response in Patients With Advanced Non-Small Cell Lung Cancer: Results of the PEMBRO-RT Phase 2 Randomized Clinical Trial. JAMA Oncol 2019;5:1276-82.

59. Buchwald ZS, Wynne J, Nasti TH, et al. Radiation, Immune Checkpoint Blockade and the Abscopal Effect: A Critical Review on Timing, Dose and Fractionation. Front Oncol 2018;8:612.

60. Steendam CMJ, Veerman GDM, Pruis MA, et al. Plasma Predictive Features in Treating EGFR-Mutated NonSmall Cell Lung Cancer. Cancers (Basel) 2020;12:3179.

61. Papadimitrakopoulou VA, Han JY, Ahn MJ, et al. Epidermal growth factor receptor mutation analysis in tissue and plasma from the AURA3 trial: Osimertinib versus platinum-pemetrexed for T790M mutationpositive advanced non-small cell lung cancer. Cancer 2020;126:373-80.

62. Tang C, Lee WC, Reuben A, et al. Immune and Circulating Tumor DNA Profiling After Radiation Treatment for Oligometastatic Non-Small Cell Lung Cancer: Translational Correlatives from a Mature Randomized Phase II Trial. Int J Radiat Oncol Biol Phys 2020;106:349-57. 
63. Gupta GP, Minn AJ, Kang Y, et al. Identifying site-specific metastasis genes and functions. Cold Spring Harb Symp Quant Biol 2005;70:149-58.

64. Uppal A, Wightman SC, Mallon S, et al. 14q32-encoded microRNAs mediate an oligometastatic phenotype. Oncotarget 2015;6:3540-52.

65. Pu Q, Huang Y, Lu Y, et al. Tissue-specific and plasma microRNA profiles could be promising biomarkers of

Cite this article as: Mentink JF, Paats MS, Dumoulin DW, Cornelissen R, Elbers JBW, Maat APWM, von der Thüsen JH, Dingemans AMC. Defining oligometastatic non-small cell lung cancer: concept versus biology, a literature review. Transl Lung Cancer Res 2021;10(7):3329-3338. doi: 10.21037/tlcr-21-265 histological classification and TNM stage in non-small cell lung cancer. Thorac Cancer 2016;7:348-54.

66. Lussier YA, Xing HR, Salama JK, et al. MicroRNA expression characterizes oligometastasis(es). PLoS One 2011;6:e28650.

67. Wu T, Dai Y. Tumor microenvironment and therapeutic response. Cancer Lett 2017;387:61-8. 